\title{
Intraductal papillary mucinous neoplasm of the pancreas in a patient with attenuated familial adenomatous polyposis
}

\author{
R Chetty, S Salahshor, B Bapat, T Berk, M Croitoru, S Gallinger
}

J Clin Pathol 2005;58:97-101. doi: 10.1136/icp.2004.020925

A 67 year old man with a clinical diagnosis of attenuated familial adenomatous polyposis (AFAP) and a past history of synchronous colon cancers in the transverse colon was also found to have an intraductal papillary mucinous neoplasm (IPMN) of the pancreas. In addition, several foci of heterotopic gastric oxyntic mucosa were noted in the duodenum, interspersed with flat and polypoid adenomas. The duodenal adenomas showed low grade dysplasia, loss of adenomatous polyposis coli (APC) protein expression, but retention of $\beta$ catenin staining, localised to the nucleus and cytoplasm. The IPMN in the pancreas showed an identical immunohistochemical profile to the duodenal adenomas. The heterotopic gastric foci in the duodenum were negative for the APC protein, and $\beta$ catenin staining was membranous in location. Although the patient did not show germline truncating APC mutations or mutations in the MYH gene, the past history, clinical features, and immunohistochemical profile of the various lesions suggest strongly that the IPMN is part of the spectrum of lesions encountered in AFAP. Whether the heterotopic oxyntic gastric mucosa in the duodenum is also related is unclear, but it may represent a forme fruste of fundic gland polyps.

$\mathrm{F}$ AP (familial adenomatous polyposis) is an autosomal dominant condition characterised by germline mutations in the APC gene on chromosome 5q21. Loss of the second allele of APC heralds the appearance of hundreds to thousands of adenomas, especially in the colorectum. Extracolonic manifestations of FAP are numerous and diverse sites are affected; however, pancreatic involvement is usually in the form of adenocarcinoma. Intraductal papillary mucinous neoplasm (IPMN) of the pancreas is a relatively recently described entity, having been first documented in 1982. ${ }^{1}$ It has been the subject of several clinicopathological, radiological, and molecular analyses. IPMN is defined as grossly visible, non-invasive, mucin producing, predominantly papillary epithelial neoplasms arising from the main pancreatic duct or branch ducts, and associated with variable distension of the associated duct. ${ }^{2}$ In addition, varying grades of cytological atypia may be encountered in the lining epithelium. IPMN is not usually associated with inherited syndromes and most cases occur sporadically. Thus far, only one case of IPMN had been encountered in a patient with FAP. ${ }^{3}$ Attenuated FAP (AFAP) is a subset of FAP that is typified by a milder clinical course, and although definitive diagnostic criteria have not been formulated, these patients have 100 or fewer adenomas, show a predilection for proximal colon involvement with rectal sparing, involvement of stomach and duodenum, and a later age of onset for both adenomas and cancer (over 40 years) than patients with FAP. ${ }^{4-6}$

\begin{abstract}
"Familial adenomatous polyposis) is an autosomal dominant condition characterised by germline mutations in the APC gene on chromosome $5 \mathrm{q} 21^{\prime \prime}$
\end{abstract}

We wish to report a patient with clinical AFAP who manifested the typical colonic and duodenal features of this syndrome, but also had IPMN of the pancreas and multiple polypoid duodenal lesions composed of fundic or oxyntic gastric mucosa, which raises the possibility of polypoid heterotopic gastric tissue or a form of fundic gland polyp.

\section{CLINICAL SUMMARY}

The patient was a 67 year old man who at the age of 60 had surgery for two synchronous colon cancers of the transverse colon. In addition, he had a family history of colon cancer: his mother had died at age 50 of colon cancer. Five years after the colectomy, the patient had several polyps removed from the region of the splenic flexure. In the course of surveillance endoscopy he was noted to have multiple duodenal polyps, which were observed over a period of time. Gastric polyps were not noted. At his last endoscopy, one of the adenomas appeared larger and was reported as showing high grade dysplasia. In view of this, the risk of another cancer and his previous history, a Whipple's procedure was planned. He is alive and well 14 months after surgery.

\section{MATERIALS AND METHODS}

The specimen was received in 10\% neutral buffered formalin. The specimen was dissected according to the departmental grossing protocol for Whipple's specimens. In addition, multiple sections from the duodenum and stomach were also taken. The entire pancreatic specimen was processed for histopathological evaluation. Sections from the specimen were processed in a routine manner and stained with haematoxylin and eosin. Immunohistochemistry was performed on formalin fixed, paraffin wax embedded tissue. In brief, $4 \mu \mathrm{m}$ sections were cut on to silane coated slides. After performing microwave antigen retrieval in $10 \mathrm{mM}$ citrate buffer (pH 6.0) in a T/T Mega microwave oven, samples were incubated with primary monoclonal mouse antibodies diluted in antibody dilution buffer (DakoCytomation, Ontario, Canada). The antibodies used and their dilutions were as follows: anti- $\beta$ catenin (1/2000; Transduction Laboratories, Boston, Massachusetts, USA), anti-APC (1/1000; Abcam Ltd, Cambridge, UK), and anti-E cadherin (1/500; Zymed, San Francisco, California, USA). As a positive control, adenomas from a known FAP case were run in parallel, and the primary antibody was omitted as a negative control. In addition, normal non-adenomatous tissues present in the stained sections were also used as positive controls and for comparative purposes, where appropriate.

Abbreviations: AFAP, attenuated familial adenomatous polyposis APC, adenomatous polyposis coli; FAP, familial adenomatous polyposis; IPMN, intraductal papillary mucinous neoplasm 


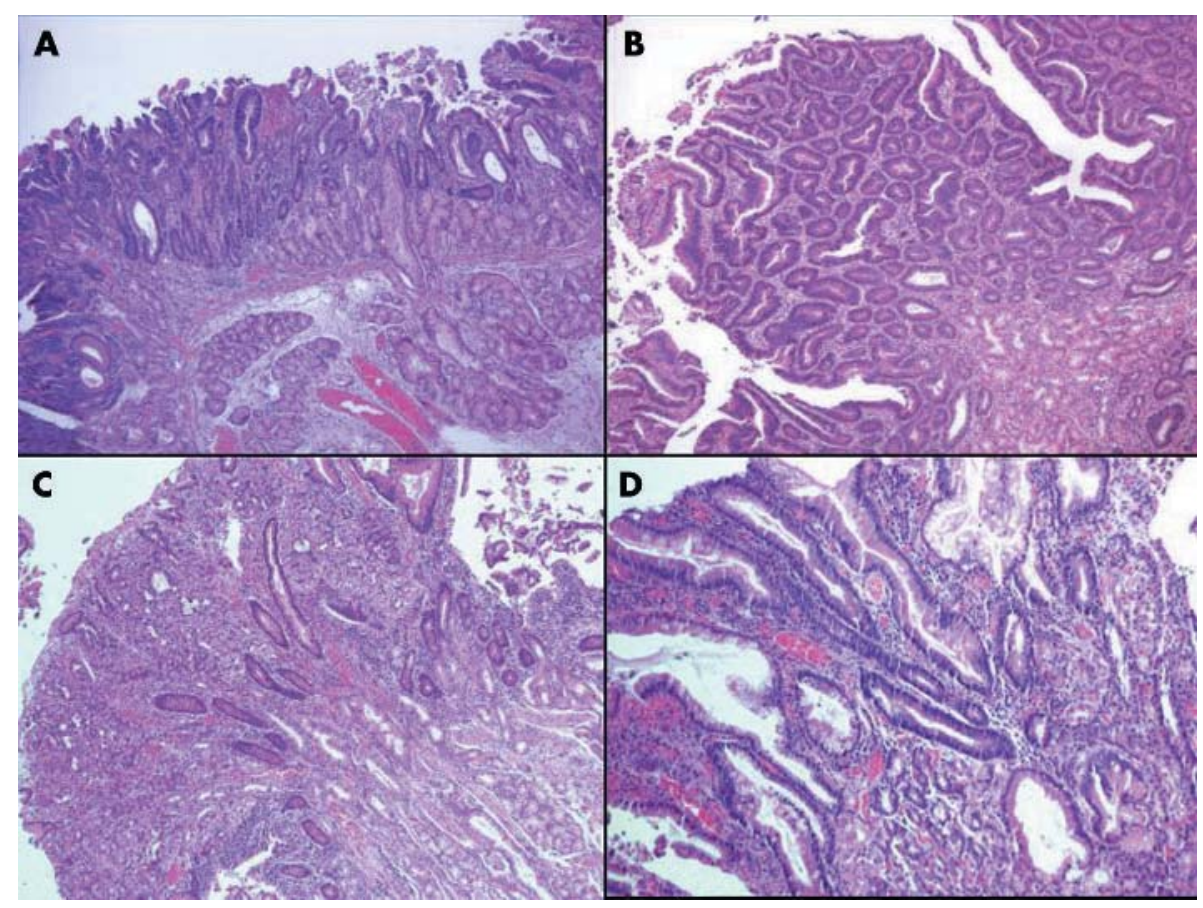

Figure 1 (A) Flat and (B) polypoid adenomas of the duodenum composed of epithelium displaying low grade dysplasia. (C) Several foci of gastric oxyntic mucosa (parietal, chief cells, and mucous cells) were noted in the duodenum. (D) Some were more polypoid in appearance with focal dilatation of glands.

The patient was also tested for germline truncating APC mutations, as is routine for screening patients for FAP and AFAP. ${ }^{6}$ Germline mutations at MYH codons 165 and 382 were tested by denaturing high performance liquid chromatography. ${ }^{7}$

\section{RESULTS}

\section{Pathological findings}

The gross specimen consisted of the distal half of the stomach $(9.0 \times 7.0 \mathrm{~cm})$, duodenum $(19.5 \mathrm{~cm}$ long $)$, and segment of the pancreas $(5.0 \times 4.0 \times 3.0 \mathrm{~cm})$. The mucosa of the

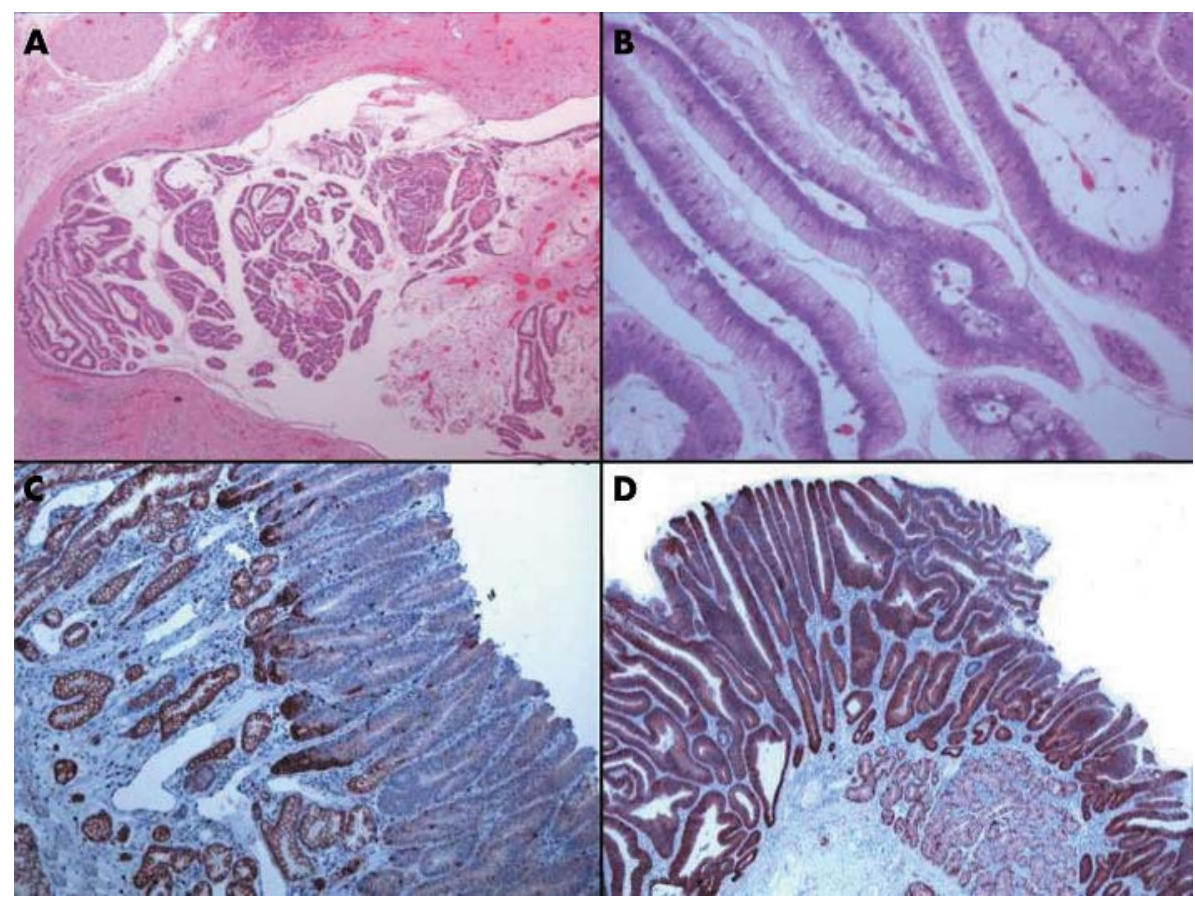

Figure 2 (A) The lesion in a branch of the main pancreatic duct was composed of papillary structures lined by intestinal-type mucosa with extravasation of mucin. (B) Under higher magnification, the epithelium showed mild to moderate dysplastic change. (C) A flat adenoma in the duodenum showing loss of staining for APC protein, whereas non-adenomatous mucosa and Brunner's glands show retention of staining. (D) In contrast, staining for the $\beta$ catenin protein is positive in the nucleus and cytoplasm of the adenomas. 
stomach was grossly unremarkable. Numerous brown/tan "roughened" or thickened areas $(0.2-0.3 \mathrm{~cm}$ in diameter, where individual foci could be identified) were noted in the first and second parts of the duodenum. The thickened area spanned $6.0 \mathrm{~cm}$ and extended to the duodenal papilla. In addition, more obvious adenomas or polyps (approximately 10) were noted in the duodenum, with the largest measuring $1.0 \mathrm{~cm}$ in maximal diameter. Within the head of the pancreas, a $1.0 \times 0.7 \mathrm{~cm}$ cystic lesion containing mucoid material was noted within a branch of the main pancreatic duct. The main pancreatic duct was not dilated and contained no lesions. The surrounding pancreatic parenchyma was normal. Fourteen regional lymph nodes were dissected.

Microscopic examination of the duodenal lesions showed the presence of numerous flat adenomas (fig 1A) and conventional tubular adenomas with a polypoid appearance (fig 1B). In most of the adenomas the lining epithelium displayed low grade dysplasia, and only a small focus of high grade dysplasia was noted in the largest tubular adenoma. However, no evidence of invasion into the lamina propria was seen. In the proximal duodenum, and interspersed between the flat adenomas, were several foci (at least 12) of gastric fundic or oxyntic type mucosa. These varied in size from small microscopic foci to more polypoid lesions (fig lC, D). They were composed of mucous glands (some of which were dilated), parietal cells, and chief cells. A similar focus was noted in the distal aspect of the stomach, close to the junction with the duodenum. These lesions were considered to be polypoid areas of gastric heterotopia. The absence of prominent dilatation militates against a definitive diagnosis of fundic gland polyps. Sections from the distal duodenum were devoid of flat adenomas, polypoid lesions, and gastric fundic gland polyps.

The lesion in a branch duct within the pancreas was papillary in configuration, caused distension of the duct, and was surrounded by sclerotic fibrous tissue (fig 2A). The lining epithelium had a distinctly intestinal-type appearance and displayed mild to moderate dysplasia (fig 2B). Extracellular mucin production was present with resultant distension of the duct housing the lesion. No invasion was present and a diagnosis of IPMN with mild to moderate dysplasia was made. The adjacent pancreatic tissue showed focal chronic pancreatitis in the region of the IPMN, secondary to duct obstruction.

\section{Immunohistochemistry}

The flat and more polypoid duodenal adenomas showed loss of APC protein immunoexpression (fig 2C). Built in positive controls (non-adenomatous duodenal mucosa and Brunner's glands) retained APC expression. In contrast, $\beta$ catenin expression was retained in the adenomas, and was strong and localised mainly to the nuclei of the adenoma cells, with some associated cytoplasmic staining (fig 2D). The heterotopic gastric foci in the duodenum also showed absence of APC protein staining but retention of $\beta$ catenin in a membrane location. The normal duodenal mucosa showed typical membrane accentuation of $\beta$ catenin staining. The IPMN had an identical staining pattern to the duodenal adenomas. Once again, APC protein expression was strikingly absent (fig 3A), whereas $\beta$ catenin was positive in both nuclear and cytoplasmic locations (fig 3B).

Staining for E cadherin was either weak or negative in the duodenal adenomas and the IPMN of the pancreas.

Molecular testing for APC mutations by the protein truncation test showed no abnormalities. The patient was wild type at MYH codons 165 and 382.

\section{DISCUSSION}

Mutations of the APC gene in FAP are now well described, as is the almost $100 \%$ penetrance associated with this condition. The APC protein regulates cytoplasmic concentrations of $\beta$ catenin by acting as a negative regulator. Sixty percent of APC mutations occur near the $\beta$ catenin binding site, resulting in the production of a truncated APC protein that has the ability to bind to, but not degrade, $\beta$ catenin. Mutations in the mutation cluster region of APC near the catenin binding sites result in the loss of tubulin binding and the inability to phosphorylate and degrade $\beta$ catenin. Hence, $\beta$ catenin protein concentrations and immunoexpression can be used as a surrogate marker to infer the status of the APC gene and its protein.

"Sixty percent of APC mutations occur near the $\beta$ catenin binding site, resulting in the production of a truncated APC protein that has the ability to bind to, but not degrade, $\beta$ catenin"

Although clinical criteria for the diagnosis of FAP have been established and are generally accepted, it has become apparent that not all patients fulfil all criteria. ${ }^{8-10}$ In 1992, Lynch and colleagues suggested that the hereditary flat adenoma syndrome may be a variant of FAP, and coined the phrase "attenuated FAP" to describe this subset of patients. ${ }^{11}{ }^{12}$ Patients with AFAP have the following features: less than 100 polyps or flat adenomas, involvement of the colon proximal to the splenic flexure with rectal sparing, duodenal adenomas, gastric fundic gland polyps, later age of onset of polyps (average age, 44 years) and colon cancer (average age, 56 years). ${ }^{4}$

The patient described in our report satisfies the clinical criteria for AFAP. In addition, he had a history of two synchronous transverse colon cancers at age 60 and his

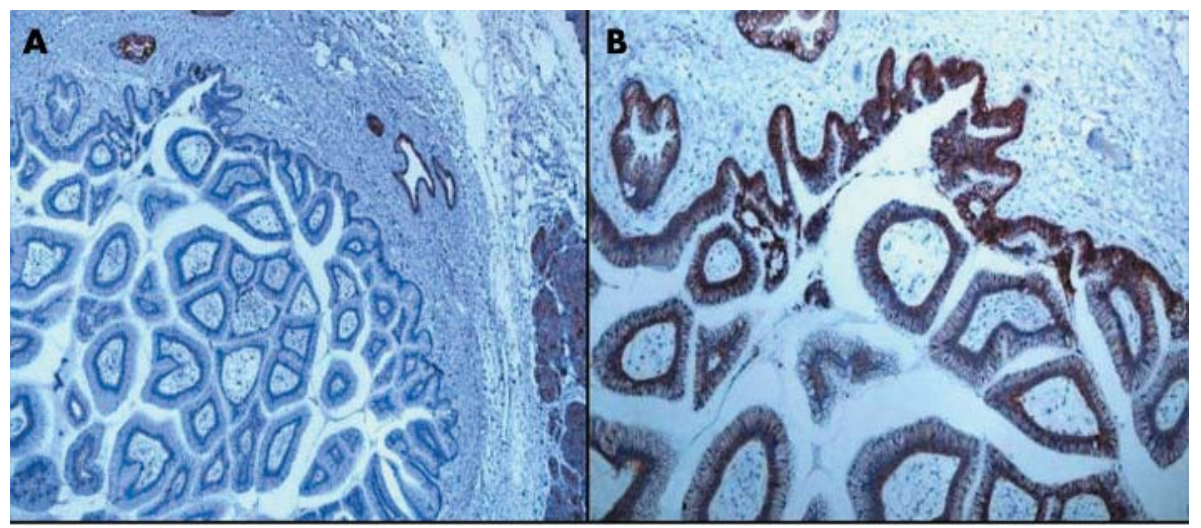

Figure 5 (A) APC protein expression is also lacking in the intraductal papillary mucinous neoplasm of the pancreas, (B) there is nuclear and cytoplasmic staining for $\beta$ catenin. 
mother died of colon cancer at age 50. The absence of an identifiable APC mutation after routine genetic screening is not surprising because in $15-50 \%$ of patients with classic FAP it is not possible to identify an APC mutation. ${ }^{513-16}$ With regard to the genetic analyses of patients manifesting the AFAP phenotype, at least 34 distinct mutations have been identified in the APC gene. ${ }^{4}$ Several mutations are sequestered at the $5^{\prime}$ end of the APC gene, within exons 3 and 4, and at the $3^{\prime}$ end of the gene. ${ }^{46}{ }^{17}$ In fact, van der Luijt and colleagues reported that deletions in the region of exon 15 at the $3^{\prime}$ end of APC do not lead to truncated APC protein production in patients with $\mathrm{AFAP},{ }^{17}$ as seen in our present patient.

Recently, a new syndrome called MYH associated polyposis has been described with a recessive pattern of inheritance as a result of biallelic missense MYH mutations. ${ }^{78}$ These patients have the phenotypic features of AFAP, including upper gastrointestinal neoplasms. ${ }^{19} 20$ Although we only tested for the two common MYH (165C and G383D) mutations, it is possible, but not likely, that this patient has one or more germline MYH mutation in other regions of the gene. Moreover, the typical recessive pattern of inheritance of MYH associated polyposis would not explain the young age of onset of colorectal cancer in the proband's mother.

The immunohistochemical findings in this patient's adenomas are in keeping with a mutation in APC. All adenomas showed complete loss or greatly decreased expression of the APC protein. This was accompanied by extremely strong immunopositivity for $\beta$ catenin. The normal duodenal mucosa showed $\beta$ catenin staining localised to the cell membrane. However, in the adenomas and the IPMN of the pancreas, strong nuclear and focal cytoplasmic staining for $\beta$ catenin was present. The lack of APC protein allows the accumulation of $\beta$ catenin. Mutations in the APC gene and alterations in the wnt signalling pathway are thought to be responsible for the nuclear localisation of $\beta$ catenin, the so called nuclear shift of the $\beta$ catenin protein. ${ }^{21}$ The nuclear shift of $\beta$ catenin staining has been noted previously in sporadic colorectal adenomas. ${ }^{22} 23$

Thus, there is immunohistochemical evidence in support of an APC gene abnormality in our case. The pancreatic IPMN showed an identical immunohistochemical profile to the duodenal adenomas. It is inferred from this that a similar pathogenetic pathway is responsible for the IPMN and the duodenal adenomas. Although pancreatic adenocarcinoma is the most common extracolonic pancreatic manifestation of FAP, a cystic papillary tumour ${ }^{24}$ and a glucagon producing endocrine tumour ${ }^{25}$ have also been described in patients with FAP. The patient with an IPMN documented by Maire et al was a 48 year old man who had a strong family history of FAP, and at the age of 33 had undergone an abdominoperineal resection for rectal carcinoma. He presented with epigastric pain and at surgery was found to have an IPMN of his main pancreatic duct. Genetic analysis showed a germline mutation in exon 15 of the APC gene, and loss of heterozygosity was seen in the pancreatic lesion, with inactivation of both alleles of APC. ${ }^{3}$

Our case had the additional feature of what we have interpreted as multiple polypoid foci of gastric heterotopia in the duodenum. These were different from the commonly encountered fundic gland polyps because mucous gland dilatation (although present focally) was not a prominent feature. However, the multiplicity of lesions, the variable size and appearance (microscopic foci to more obvious polypoid lesions), their location interspersed between flat and polypoid adenomas in the duodenum, and the clinical scenario all favour these lesions being associated with the clinical syndrome of AFAP. Perhaps the slightly different appearance compared with typical fundic gland polyps results from the
Take home messages

- We report a case of attenuated familial adenomatous polyposis (AFAP) together with intraductal papillary mucinous neoplasm (IPMN) of the pancreas

- In addition, foci of heterotopic gastric oxyntic mucosa were noted in the duodenum, interspersed with flat and polypoid adenomas

- On the basis of the immunohistochemical staining patterns for the adenomatous polyposis coli and $\beta$ catenin proteins and the clinical scenario, we believe that the duodenal adenomas and IPMN of the pancreas are pathogenetically related, with the IPMN being an unusual extracolonic manifestation of FAP and/or AFAP syndromes

- Whether the polypoid oxyntic gastric mucosa is part of the spectrum of lesions is more difficult to prove conclusively

yet to be identified genetic abnormality of APC in our patient. Multiple fundic gland polyps have been described in the duodenal bulb in a patient without intestinal polyps, ${ }^{26}$ but this appears to be the only reported case in the English literature of such an occurrence. The absence of APC protein expression and the normal staining and distribution of $\beta$ catenin are in keeping with the study by Abraham et al, who found no $\beta$ catenin gene abnormalities in FAP associated fundic gland polyps. ${ }^{27}$ Furthermore, an immunohistochemical analysis of syndromic and sporadic fundic gland polyps showed loss of APC protein expression in syndromic polyps, whereas aberrant nuclear localisation of the $\beta$ catenin protein was not found in either syndromic or sporadic fundic gland polyps. ${ }^{28}$ Thus, there is evidence that the so called fundic gland polyps encountered in the duodenum in our case conform immunohistochemically to the syndromic fundic gland polyps described by Hassan et al. ${ }^{28}$

We wish to highlight an unusual extracolonic manifestation in AFAP, that of IPMN in the pancreas and polypoid oxyntic gastric heterotopia (with a superficial resemblance to fundic gland-type polyps) in the duodenum. On the basis of the immunohistochemical staining patterns for the APC and $\beta$ catenin proteins and the clinical scenario, we believe that the duodenal adenomas and IPMN of the pancreas are pathogenetically related, with the IPMN being an unusual extracolonic manifestation of FAP and/or AFAP syndromes. Whether the polypoid oxyntic gastric mucosa is part of the spectrum of lesions is more difficult to prove conclusively.

\section{Authors' affiliations}

R Chetty, Department of Pathology, Department of Laboratory Medicine and Pathobiology, University of Toronto and University Health Network, Toronto, ON M5G 2M9, Canada

S Salahshor, Department of Medical Biophysics, Ontario Cancer Institute T Berk, Familial GI Cancer Registry, Samuel Lunenfeld Research Institute, Mount Sinai Hospital, Toronto, Ontario M5G 1X5, Canada

B Bapat, M Croitoru, Center for Cancer Genetics, Samuel Lunenfeld Research Institute, Mount Sinai Hospital

S Gallinger, Department of Surgery, University of Toronto and University Health Network, Toronto, Canada

Correspondence to: Dr R Chetty, University Health Network, Princess Margaret Hospital, 610 University Avenue, Fourth Floor, Suite 302, Room 312, Toronto, ON M5G 2M9, Canada; runjan.chetty@uhn.on.ca

Accepted for publication 1 September 2004 


\section{REFERENCES}

1 Ohhashi K, Murakami M, Murayama M, et al. Four cases of mucous secreting pancreatic cancer. Prog Dig Endosc 1982;20:348-51.

2 Klimstra DS, Takaori K, Hruban RH, et al. Consensus criteria for the classification of pancreatic intraepithelial neoplasia (PanIN) and intraductal papillary mucinous neoplasms (IPMNs). Mod Pathol 2004;17:305A.

3 Maire F, Hammel P, Terris B, et al. Intraductal papillary and mucinous pancreatic tumour: a new extracolonic tumour in familial adenomatous polyposis. Gut 2002;51:446-9.

4 Hernegger GS, Moore HG, Guillem JG. Attenuated familial adenomatous polyposis. An evolving and poorly understood entity. Dis Colon Rectum 2002;45: 127-36

5 Knudsen AL, Bisgaard AL, Bulow S. Attenuated familial adenomatous polyposis (AFAP). A review of the literature. Familial Cancer 2003:2:43-55.

6 Soravia C, Berk T, Madlensky L, et al. Genotype-phenotype correlations in attenuated adenomatous polyposis coli. Am J Hum Genet 1998;62:1290-301

7 Al-Tassan N, Chmiel NH, Maynard J, et al. Inherited variants of MYH associated with somatic $\mathrm{G}: \mathrm{C} \rightarrow \mathrm{T}: \mathrm{A}$ mutations in colorectal tumors. Nat Genet 2002;30:227-32.

8 Lynch HT, Smyrk T, Lanspa S, et al. Flat adenomas in a colon cancer-prone kindred. J Natl Cancer Inst 1988;80:278-282.

9 Lynch HT, Smyrk T, Lanspa S, et al. Phenotypic variation in colorectal adenoma/cancer expression in two families. Hereditary flat adenoma syndrome. Cancer 1990;66:909-15.

10 Leppert M, Burt R, Hughes J, et al. Genetic analysis of a predisposition to colon cancer in a family with a variable number of adenomatous polyps. N Engl J Med 1990;322:904-8.

11 Lynch HT, Smyrk T, Watson P, et al. Hereditary flat adenoma syndrome: a variant of familial adenomatous polyposis? Dis Colon Rectum 1992;35:411-21.

12 Lynch HT, Smyrk T, McGinn T, et al. Attenuated familial adenomatous polyposis (AFAP): phenotypically and genotypically distinctive variant of FAP Cancer 1995; 76:2427-33

13 Moisio A-L, Jarvinen H, Peltomaki P. Genetic and clinical characterization of familial adenomatous polyposis: a population based study. Gut 2002;50:845-50

14 Heinimann K, Mullhaupt B, Weber W, et al. Phenotypic differences in familial adenomatous polyposis based on APC gene mutation status. Gut 1998;43:675-9.
15 van der Luijt RB, Khan PM, Vasen HF, et al. Molecular analysis of the APC gene in 105 Dutch kindreds with familial adenomatous polyposis: 67 germline mutations identified by DGGE, PTT and Southern analysis. Hum Mutat 1997;9:7-16

16 Powell SM, Petersen GM, Krush AJ, et al. Molecular diagnosis of familial adenomatous polyposis. N Engl J Med 1993;329:1982-7.

17 van der Luijt RB, Khan PM, Vasen HF, et al. Germline mutations in the 3' part of APC exon 15 do not result in truncated proteins and are associated with familial adenomatous polyposis coli. Hum Genet 1996;98:727-34.

18 Jones S, Emmerson P, Maynard J, et al. Biallelic germline mutations in MYH predispose to multiple colorectal adenoma and somatic $\mathrm{G}: \mathrm{C} \rightarrow \mathrm{T}: \mathrm{A}$ mutations. Hum Mol Genet 2002;11:2961-7.

19 Sieber OM, Lipton L, Crabtree M, et al. Multiple colorectal adenomas, classical adenomatous polyposis and germ-line mutations in MYH. N Engl J Med 2003;348:791-9.

20 Sampson JR, Dolwani S, Jones S, et al. Autosomal recessive colorectal adenomatous polyposis due to inherited mutations in MYH. Lancet 2003;362:39-41.

21 Peifer M. Beta-catenin as oncogene: the smoking gun. Science 1997:275:152-3.

22 Hao X, Tomlinson I, llyas $M$, et al. Reciprocity between membranous and nuclear expression of beta-catenin in colorectal tumours. Virchows Arch 1997:431:167-72

23 Valizadeh A, Karayiannakis AJ, el-Hariry I, et al. Expression of E-cadherinassociated molecules (alpha-, beta-, and gamma-catenins and p120) in colorectal polyps. Am J Pathol 1997;150:1977-84.

24 Le Borgne J, Bouvier S, Fiche $M$, et al. Cystic and papillary tumor of the pancreas: diagnostic and developmental uncertainties. Apropos of a case [ln French]. Chirurgie 1997; 122:31-4.

25 Stewart CJ, Imrie CW, Foulis AK. Pancreatic islet cell tumour in a patient with familial adenomatous polyposis. J Clin Pathol 1994;47:860-1.

26 Zygulska A, Goszcz A, Bogdal J, et al. Multiple fundic gland polyps in duodenal mucosa not associated with colonic polyposis. Pol J Patho 1997:48:201-3.

27 Abraham SC, Nobukawa B, Giardiello FM, et al. Sporadic fundic gland polyps. Common gastric polyps arising through activating mutations in the $\beta$ catenin gene. Am J Pathol 2001;158:1005-10

28 Hassan A, Yerian LM, Kuan S-F, et al. Immunohistochemical evaluation of adenomatous polyposis coli, b-catenin, c-myc, cyclin D1, p53, and retinoblastoma protein expression in syndromic and sporadic fundic gland polyps. Hum Pathol 2004;35:328-34. 